\title{
CanariCam: a multimode mid-infrared camera for the Gran Telescopio CANARIAS
}

Charles M. Telesco, David Ciardi, James French, Christ Ftaclas, Kevin T. Hanna, et al.

Charles M. Telesco, David Ciardi, James French, Christ Ftaclas, Kevin T. Hanna, David B. Hon, James H. Hough, Jeffrey Julian, Roger Julian, Mark Kidger, Chris C. Packham, Robert K. Pina, Frank Varosi, R. Glenn Sellar, "CanariCam: a multimode mid-infrared camera for the Gran Telescopio CANARIAS," Proc. SPIE 4841, Instrument Design and Performance for Optical/Infrared Ground-based Telescopes, (7 March 2003); doi: 10.1117/12.458979

Event: Astronomical Telescopes and Instrumentation, 2002, Waikoloa, Hawai'i, United States 


\title{
CanariCam: a multimode mid-infrared camera for the Gran Telescopio CANARIAS
}

\author{
C. M. Telesco*a, D. R. Ciardi ${ }^{\mathrm{a}}$, J. French ${ }^{\mathrm{a}}$, C. Ftaclas ${ }^{\mathrm{b}}$, K. T. Hanna ${ }^{\mathrm{a}}$, D. B. Hon ${ }^{\mathrm{a}}$, J. H. Hough ${ }^{\mathrm{c}}$, \\ J. Julian ${ }^{a}$, R. Julian, ${ }^{\mathrm{a}}$, M. Kidger ${ }^{\mathrm{d}}$, C. Packham ${ }^{\mathrm{a}}$, R. K. Piña ${ }^{\mathrm{a}}$, G. Sellar ${ }^{\mathrm{e}}$, F. Varosi ${ }^{\mathrm{a}}$ \\ ${ }^{\mathrm{a}}$ Department of Astronomy, University of Florida; ${ }^{\mathrm{b}}$ Institute for Astronomy, University of Hawaii; \\ ${ }^{\mathrm{c}}$ University of Hertfordshire, UK; ${ }^{\mathrm{d}}$ Instituto de Astrofisica de Canarias, Spain; \\ ${ }^{\mathrm{e}}$ Florida Space Institute, University of Central Florida
}

\begin{abstract}
The University of Florida is developing a mid-infrared camera for the 10.4-meter Gran Telescopio CANARIAS. CanariCam has four science modes and two engineering modes, which use the same 320 x 240-pixel, arsenic-doped silicon, blocked-impurity-band detector from Raytheon. Each mode can be remotely selected quickly during an observing sequence. The pixel scale is 0.08 arcsec, resulting in Nyquist sampling of the diffraction-limited point-spreadfunction at $8 \mu \mathrm{m}$, the shortest wavelength for which CanariCam is optimized. The total available field of view for imaging is $26 \operatorname{arcsec} \times 19$ arcsec. The primary science mode will be diffraction-limited imaging using one of several available spectral filters in the $10 \mu \mathrm{m}(8-14 \mu \mathrm{m})$ and $20 \mu \mathrm{m}(16-25 \mu \mathrm{m})$ atmospheric windows. Any one of four plane gratings can be inserted for low and moderate-resolution $(R=100-1300)$ slit spectroscopy in the 10 and $20-\mu \mathrm{m}$ regions. Insertion of appropriate field and pupil stops converts the camera into a coronagraph, while insertion of an internal rotating half-wave plate, a field mask, and a Wollaston prism converts the camera into a dual-beam polarimeter.
\end{abstract}

Keywords: Gran Telescopio CANARIAS, infrared imaging, spectroscopy, polarimetry, coronagraphy, BIB detectors

\section{OVERVIEW}

CanariCam is a state-of-the-art multimode camera being developed at the University of Florida for use at the Gran Telescopio CANARIAS (GTC). CanariCam will become operational in Fall 2003 and will be available for the GTC Day-1 science observing shortly thereafter. This paper describes the camera and its key observational modes. CanariCam's electronics are nearly identical to those of T-ReCS, the Gemini South mid-infrared camera, and the reader is referred to Telesco et al. ${ }^{1}$ for a more detailed description of them. CanariCam is optimized for use at $8-25 \mu \mathrm{m}$, the so-called mid-infrared spectral region, but it is useful for certain key engineering observations down to about $2 \mu \mathrm{m}$. The goal has been to provide the GTC astronomical community with an outstanding "workhorse" multi-mode instrument for use in the atmospheric windows near $10 \mu \mathrm{m}$ (roughly spanning 8 to $14 \mu \mathrm{m}$ ) and $20 \mu \mathrm{m}$ (roughly spanning 16 to $25 \mu \mathrm{m}$ ). The detector is an arsenic-doped silicon, blocked-impurity-band (BIB, or IBC) device from Raytheon, with peak QE in the $8-25 \mu \mathrm{m}$ region and a rapid decrease in QE at longer wavelengths. In addition to the selection of this particular detector device, optimisation for the mid-IR has entailed: (1) matching the plate scale of the instrument to the $50 \mu \mathrm{m}$ detector pixel size so that the point-spread-function (PSF) is Nyquist-sampled at $8 \mu \mathrm{m}$, (2) selecting various window, filter, and coating materials that maximize throughput at these wavelengths, (3) designing the electronics so that the detector can be read out rapidly enough not to saturate in the high-thermal background that is characteristic of the midinfrared regime, and (4) designing the cryostat to operate below $10 \mathrm{~K}$ and minimize background radiation from all extraneous sources. Outstanding image quality, high throughput, and excellent mechanical stability are critical characteristics of CanariCam. These properties must be achieved in the context of operational convenience, long-term reliability, and reasonable development and maintenance costs. As a facility workhorse instrument, CanariCam must possess a special combination of simplicity and outstanding performance. CanariCam will meet or exceed these expectations, and it is expected to be friendly to the GTC technical staff that must support it.

CanariCam will address a broad range of scientific problems. Astronomical bodies at temperatures of 100-1000 K emit significant mid-IR radiation. Of particular importance are the ubiquitous small particles-dust-that absorb radiation at virtually any wavelength and transform it into infrared, sub-millimeter, or millimeter radiation. Mid-infrared continuum emission from the dust is diagnostic of the properties of a great variety of astrophysical objects, including planets, 
circumstellar disks, star-forming regions, and starburst and active galactic nuclei. With multi-wavelength mid-infrared imaging, one can locate energy sources that power often enormous luminosities, trace the distributions of dust particles and their temperatures, and determine how UV and optical radiation, which heats the dust, propagates throughout the infrared-emitting regions. The spectroscopic mode will permit the exploration of a broad range of solid-state and gas emission features. The coronagraphic mode is ideally suited to the investigation of sub-stellar objects and circumstellar material in close proximity to 'parent' stars. Finally, polarimetric observations allow detailed mapping of the magnetic alignment of dust particles in objects such as circumstellar disks, young stars, and active galaxies. Each mode of operation is discussed in detail below.

\section{BASIC CONFIGURATION AND OPTICAL DESIGN}

The CanariCam optics and detector are encased in a hexagonally shaped dewar to which is attached a coldhead capable of cooling the detector to its operating temperature near $8 \mathrm{~K}$ and the optics and interior hardware to appropriately low temperatures to minimize thermal background. No liquid cryogens are used in CanariCam. The CanariCam instrument is illustrated in Fig. 1. Note particularly the window-turret, which allows one to select in real time from among a number of entrance windows to permit optimization of the window material for specific science goals and observing conditions. As discussed further below, the detector and all optical components and associated mechanisms are attached to the rigid internal optical bench, which is thermally isolated from the radiation shields and outer case by several bands, or cylinders, of G-10 fiberglass standoffs. All of CanariCam's powered optics used in the science modes are reflective. The imaging, spectroscopic, and coronagraphic modes are fully reflective except for the entrance window and filters. The polarimetric mode employs a half-wave plate and a Wollaston prism, both of which are transmissive. The nearly allreflective design is achromatic and minimizes scattering and straylight. Except for the entrance window, all of the optical components and associated mechanisms are attached to the optical bench. These optics and mechanisms are distributed on either side of the bench, with a fold that diverts the beam through the bench. In the following description we refer to Fig. 2, which shows the optical bench and the associated optical components.

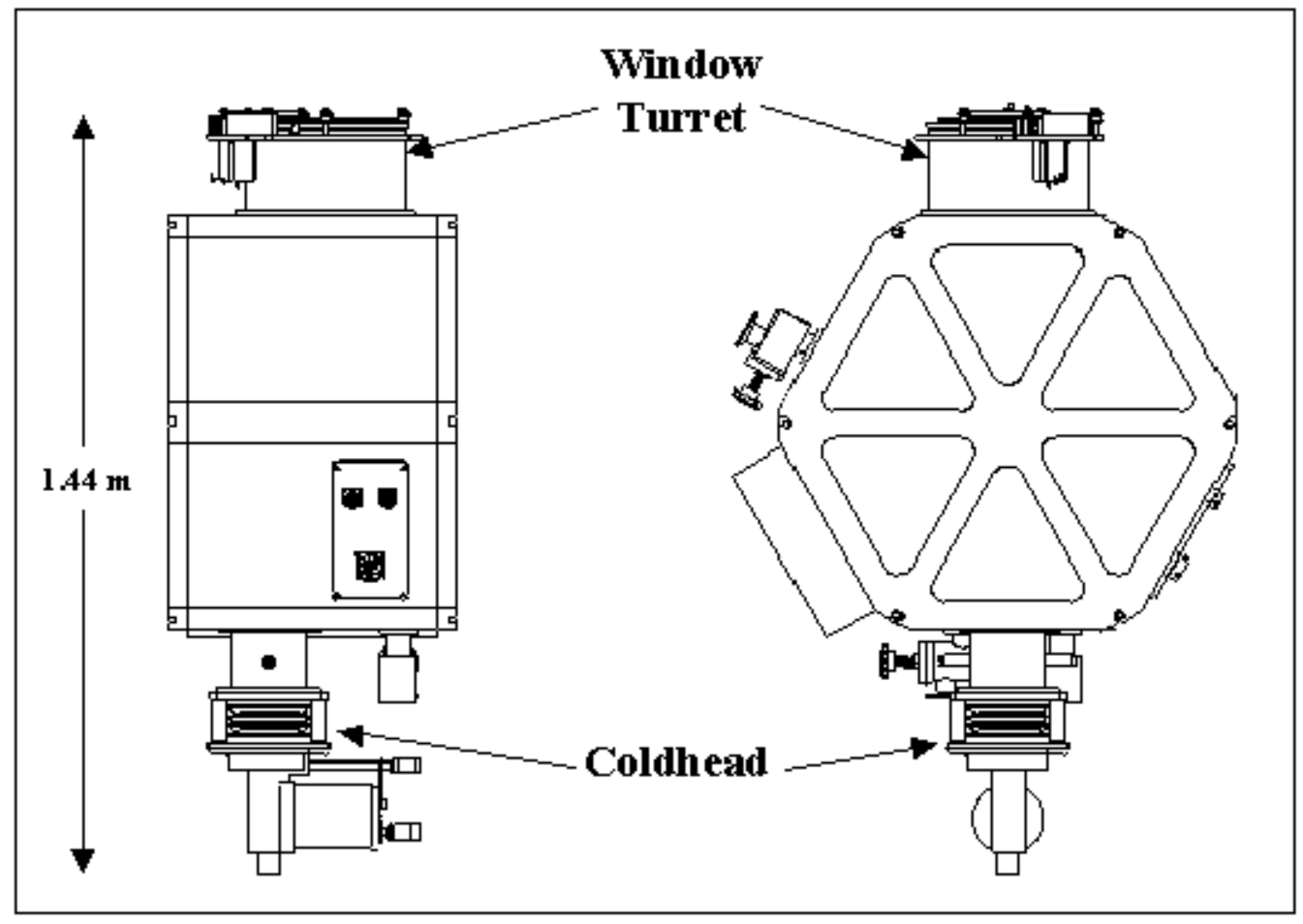

Fig. 1: The CanariCam enclosure, illustrating its overall shape, scale, and locations of the coldhead and window turret. 


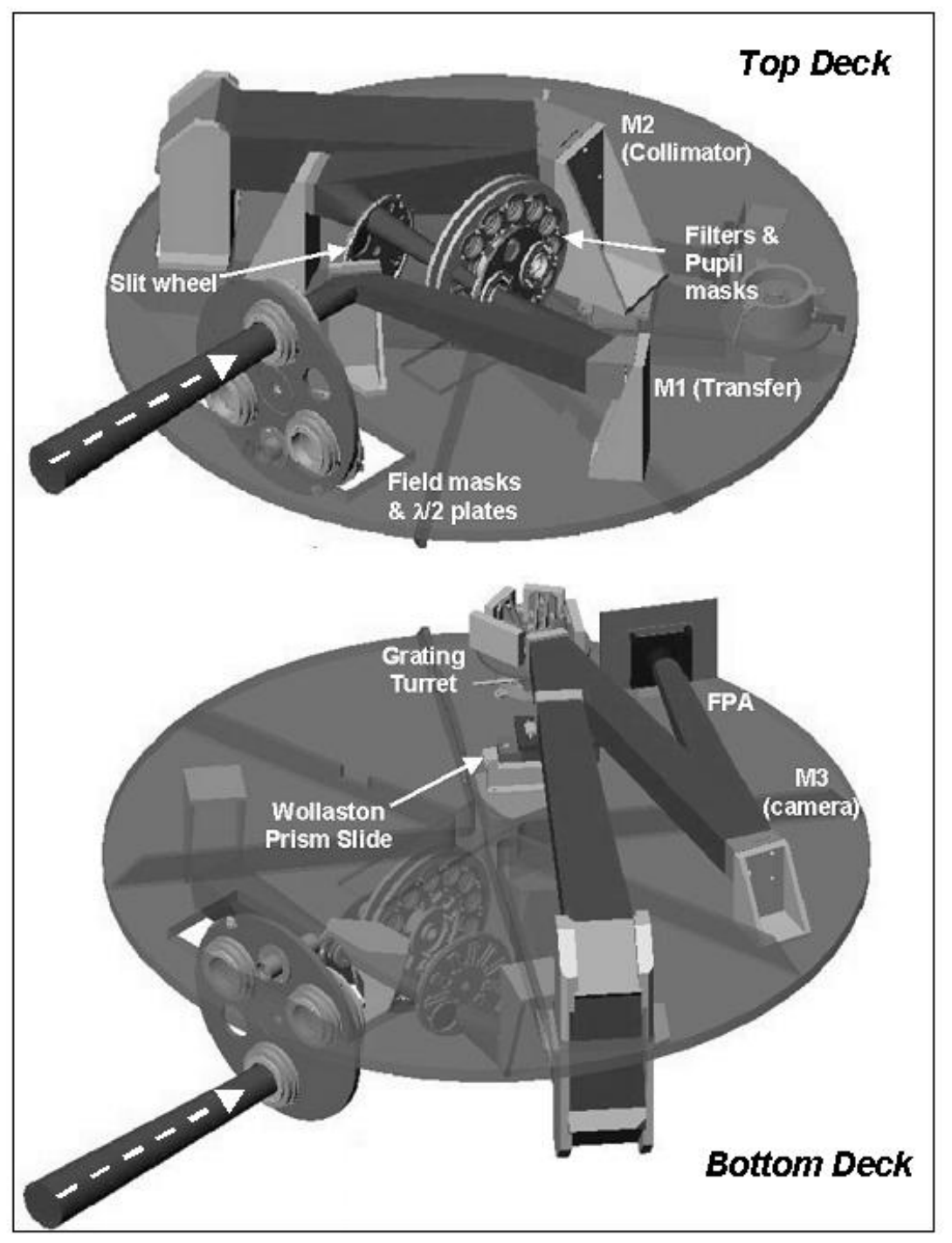

Fig. 2: The CanariCam optical layout shown attached to the optical bench

The telescope beam passes through the dewar entrance window (also the pressure window) and comes to a focus inside the dewar. Between the entrance window and the telescope focal plane, half-wave plates for the polarimetric mode or a lens assembly for the window-imaging mode can be inserted. The half-wave plates can be rotated to four position angles. A selection of aperture stops (including occulting masks for coronagraphy and polarimetry) is installed in a wheel at the location of the telescope focus. The diverging beam is then incident on the powered transfer mirror M1, which forms an image of the telescope pupil and re-images the telescope focal plane. Pupil stops are on a rotating assembly at the pupil image, and a double filter wheel is very close to this position. The rotating pupil-stop assembly permits rotation of the complex pupil mask in order to provide maximum throughput and straylight rejection. One can insert a dual-lens assembly in this region in order to image the telescope pupil image onto the detector. A slit wheel permits insertion at the re-imaged telescope focal plane an open hole for imaging or a selection of long narrow slits for spectroscopy. The beam is then incident on the collimator M2, which forms a pupil image near the position where the gratings (for spectroscopy) and a flat mirror (for all other modes) are mounted on a turret. A Wollaston prism can be inserted into the collimated beam between M2 and the turret. After the turret the beam is incident on the camera mirror M3, which images the astronomical field onto the detector array. 


\subsection{Imaging}

\section{MODES}

Mid-infrared imaging is considered to be the fundamental science mode for CanariCam, and implementation of the other modes cannot compromise the imaging performance. The key image-quality requirement is that the energy enclosed within a square of pixels at the final image shall be at least $80 \%$ of that expected for the telescope alone at a wavelength of $8 \mu \mathrm{m}$. Even at this wavelength, the most abberrated part of the image plane delivers an enclosed energy that is $96 \%$ of the diffraction limit, corresponding to a Strehl ratio of $92 \%$. The enclosed energies and Strehl ratios are higher for all other fields. The distortion of the field at the corner relative to the center is approximately 1 pixel. To achieve the highest possible throughput for a given set of observing conditions, the ferrofluidic window turret permits one to select from several entrance windows. For observing only in the $10-\mu \mathrm{m}$ atmospheric window, a $\mathrm{ZnSe}$ window is used that has a transmittance greater than $94 \%$, but is opaque in the $20 \mu \mathrm{m}$ window. For observing in both the 10 and $20-\mu \mathrm{m}$ windows, a $\mathrm{KBr}$ window, for which the transmittance is greater than $92 \%$, can be used. However, $\mathrm{KBr}$ is water-soluble, and cannot be used under higher-humidity conditions. Under such conditions a KRS-5 window will be used, albeit with a loss in throughput (transmission $>70 \%$ at 2-35 $\mu \mathrm{m}$ ). The throughput of the optical system (windows and mirrors only) is anticipated to be $90 \%$ with the $\mathrm{ZnSe}$ window and better than $85 \%$ with the $\mathrm{KBr}$ window. Including the detector and filters, the total system throughput in the imaging mode is expected to be $>21 \%$.

\subsection{Spectroscopy}

The spectrometer follows that of the Czerny-Turner layout using any one of four classical plane gratings installed on a turret. The diameter of the pupil image near the grating has been maximized to achieve moderate spectral resolution, with the constraint that the system must fit within the envelope at the folded Cassegrain focus. The folded cassegrain station is the most constraining of the three focal-plane envelopes (cassegrain, nasmyth, and folded cassegrain) where CanariCam must be deployable. The resultant pupil image on the grating turret folding flat mirror is $24.4 \mathrm{~mm}$ in diameter. The general grating properties are listed in Table 1. The gratings are optimized for use in first order, although use in other orders will be permitted. Substrates will be aluminum and coated with protected gold. CanariCam will employ a slit wheel mechanism that permits the selection of any of nine slits with the use of an upstream 'dekker' (i.e. oversized slit) in the aperture wheel. One will then be able to match the slit width to the seeing conditions or spectral resolution requirements during the observing session itself.

Table 1: Nominal Grating Properties

\begin{tabular}{|l|l|l|l|l|}
\hline Characteristic & $\mathbf{1 0} \boldsymbol{\mu} \mathbf{m}$ LoRes & $\mathbf{2 0} \mu \mathbf{m}$ LoRes & $\mathbf{1 0} \boldsymbol{\mu m}$ HiRes & $\mathbf{2 0} \boldsymbol{\mu m}$ HiRes \\
\hline Central $\boldsymbol{\lambda} \mathbf{c}(\boldsymbol{\mu m})$ & 10.5 & 20.5 & 10.5 & 20.5 \\
\hline Blaze $\boldsymbol{\lambda}(\boldsymbol{\mu \mathbf { m } )}$ & 9.87 & 19.96 & 10.08 & 20.18 \\
\hline Blaze Angle $(\mathbf{d e g})$ & 2.6 & 4.0 & 16.4 & 20.8 \\
\hline Lines $/ \mathbf{m m}$ & 9.1 & 6.1 & 56.0 & 35.2 \\
\hline $\boldsymbol{\Delta} \boldsymbol{\lambda}(\boldsymbol{\mu m})$ & 6 & 9 & 0.98 & 1.54 \\
\hline Angle of incidence $(\mathbf{d e g})$ & 14.8 & 15.7 & 29.5 & 33.7 \\
\hline Angle of diffraction $(\mathbf{d e g})$ at $\boldsymbol{\lambda} \mathbf{c}$ & 9.2 & 8.3 & 5.5 & 9.7 \\
\hline $\mathbf{R}=\boldsymbol{\lambda} / \boldsymbol{\Delta} \boldsymbol{\lambda}$ & 175 & 120 & 1313 & 891 \\
\hline Smallest resolvable $\boldsymbol{\Delta} \boldsymbol{\lambda}(\boldsymbol{\mu m})$ & 0.06 & 0.17 & 0.008 & 0.023 \\
\hline
\end{tabular}

\subsection{Polarimetry}

CanariCam has a dual-beam polarimetric mode for the $10 \mu \mathrm{m}$ spectral region, with extension to the $20 \mu \mathrm{m}$ region currently being considered. This design permits simultaneous measurement of the ordinary $(o)$ and extraordinary $(e)$ rays, which not only increases observational efficiency but also minimizes effects of seeing and changes in atmospheric transparency. For a dual-beam polarimeter, an absolute uncertainty in the degree of polarization of $0.5 \%$ requires a $\mathrm{S} / \mathrm{N}$ ratio of $\sim 300: 1$ in total flux. For the source-limited case this corresponds to $8 \times 10^{4}$ photons or $4 \times 10^{4}$ per Stokes parameter. Thus, with a dual-beam system the accuracy obtained is a function of photon numbers only, and accurate polarimetry of bright sources can be carried out during observing conditions that are too bad for almost any other type of quantitative observation. CanariCam's polarimetric mode will be able to measure degrees of polarization as small as $\sim 0.1 \%$. The key components of the polarimetric design are: (1) a cooled, rotatable, sulphur-free CdSe composite zeroorder half-wave plate (HWP, retarder) within the cryostat located just upstream from the telescope focal plane; (2) a 
focal-plane mask at the telescope focal plane; and (3) a sulphur-free air-gap CdSe Wollaston prism (analyzer) located just upstream from the grating turret. The HWP (one for the $10-\mu \mathrm{m}$ window and one under consideration for the $20-\mu \mathrm{m}$ window) will be rotated sequentially to four different (detent-determined) discrete orientations $(0,22.5,45$, and 67.5 degrees), with images being taken at each HWP orientation. The Wollaston prism is inserted into the beam on a slide, and produces an angular separation between the orthogonally polarized states, thus producing two beams, the so-called $o$ and $e$ rays. Hence, two images of the object are formed on the detector.

Because the HWP retarder is the first optical component in the CanariCam system, the instrumental polarization (IP) is negligible. Any amount of IP biases the measured the polarization, but for most telescopes it is usually $\leq 0.05 \%$ at the unfolded Cassegrain focus. Typically a 45 degree reflection, as occurs with a Nasmyth focus, produces a polarization of $\sim 5 \%$ in the visible, $0.5 \%$ in the near-infrared, and less in the mid-IR, although the actual values depend on the nature and cleanliness of the reflecting surfaces and may change with time. As long as the IP is small, it is added vectorally to the Stokes vector and can therefore be subtracted in the same way. For large IP and large source polarization, a full Mueller matrix treatment is needed. The IP can be determined by observation of unpolarized stars and assuming, as is the case at mid-infrared wavelengths, that it is only a few tenths of a percent. The effect of oblique reflections prior to the modulator should then be small.

When using a dual-beam analyzer, a focal plane mask such as that shown in Fig. 3 is required so that extended objects can be observed without overlap of the orthogonally polarized images. The separation of beams is usually a compromise between possible optical aberrations produced for large separations and cross-talk for too small a separation. Large separations are convenient, since extended objects may be fully covered by one of the mask gaps, and observations can be made with a single setting of the telescope.

\subsection{Coronagraphy}

For stars observed at mid-infrared wavelengths, thermal background emission from the sky and the telescope will be many orders of magnitude larger than the stellar flux. By the use of chopping and nodding techniques, however, it is possible to remove this background at levels approaching one part in a million. Once the background has been removed, the focal plane intensity will be dominated by the stellar point spread function (PSF), the wings of which can be thought of as a "halo" arising from diffracted and scattered light. The key motivation for the coronagraphic mode is to suppress

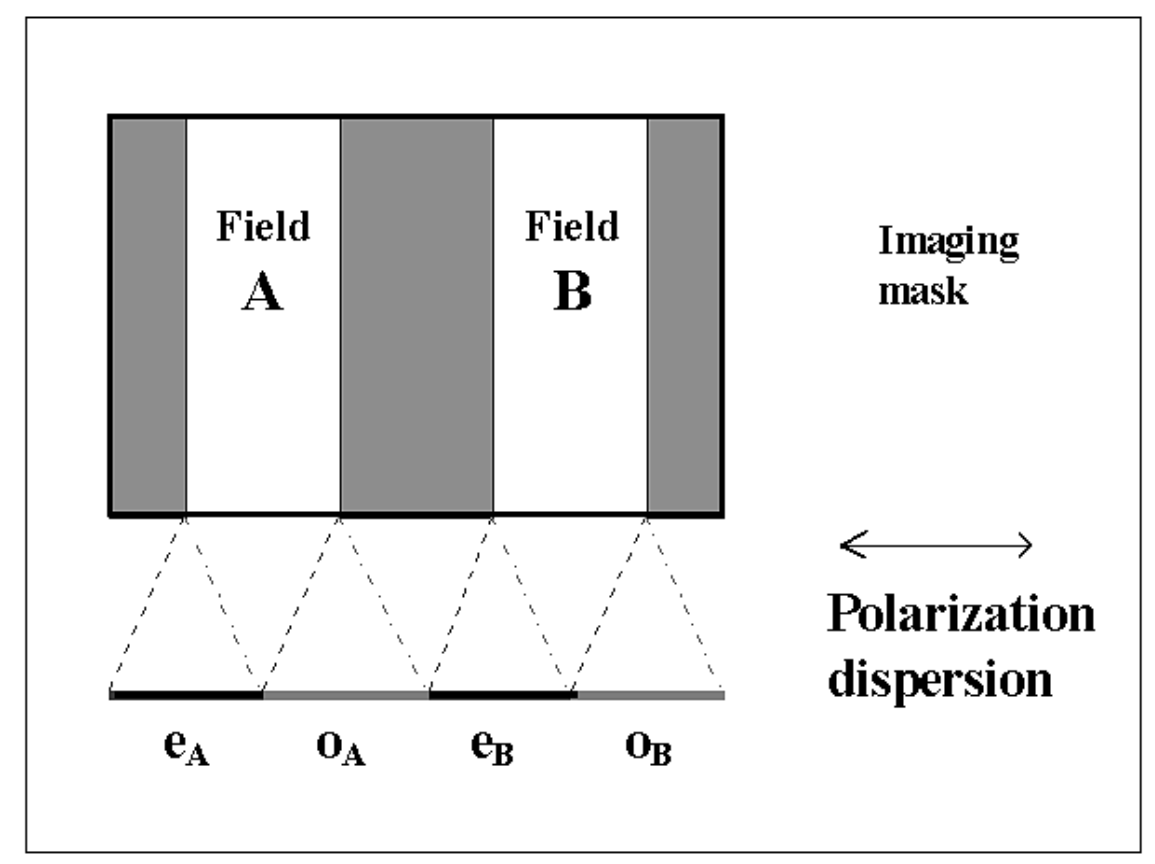

Fig. 3: Illustrative mask design for the CanariCam polarimetry mode. 
the stellar halo, or PSF wings, to allow circumstellar searches for disks and faint companions. An important goal of an effective coronagraphic mode is to minimize residual diffractive structure in the focal plane with minimum losses in field of view and throughput.

To illustrate key features of the CanariCam coronagraphic mode, we consider the case of good atmospheric conditions at an observing wavelength of $10 \mu \mathrm{m}$ defined by a Fried scale length of order the size of the aperture $(10 \mathrm{~m})$ and an outer scale twice the size of the aperture $(20 \mathrm{~m})$. These parameters define peak atmospheric conditions. The shape of the GTC entrance pupil with the model atmosphere is shown in Fig. 4. We assume that the star is occulted in the telescope focal plane by a hard-edged (top-hat), low reflectivity circular mask occulting mask 0.83 arcsec in radius. In Fig. 5 we show a plot of the log of the azimuthally averaged intensities as a function of the distance from the star. The top line represents the stellar image PSF for no coronagraphic masks. The line denoted "F2 circle-no center" represents the stellar image for the occulting mask and a circular stop inscribed in the hexagonal pupil. The line denoted "F2-circles" represents the stellar image for the occulting mask combined with a pupil mask consisting of a inscribed circle and a central circular mask that blocks the central pupil obscuration. Finally, the line denoted "F2 Serrated" represents the stellar image for the occulting mask combined with a pupil mask that has a serrated and hex-shaped outer mask that matches the pupil shape and masks that block the secondary-mirror spiders.

We find that the occulting spot combined with even the simplest circular pupil mask (which does not mask the central secondary-mirror structure) provides substantial gains in the rejected light; the azimuthally averaged suppression ratio is about four. However, by applying a central mask in the pupil stop to block the pupil obscuration, one achieves a suppression ratio of about an order of magnitude. The azimuthal averages suggest that the pupil mask with two circles(no spider mask) and the pupil mask that is fully matched to the serrated pupil (including the central obscuration and the spiders) provide comparable performance. At first sight, the 2-circle mask appears more advantageous, since it would not have to be rotated as the pupil rotates. However, the azimuthal average does not show certain crucial information. When we look at the detailed focal plane intensity distribution, there are large regions of the focal plane where the spider masking reduces the background by an order of magnitude or more better than the case with no spider masking.

Given this conclusion we have developed a design that employs a rotating pupil stop and that maximizes the throughput.
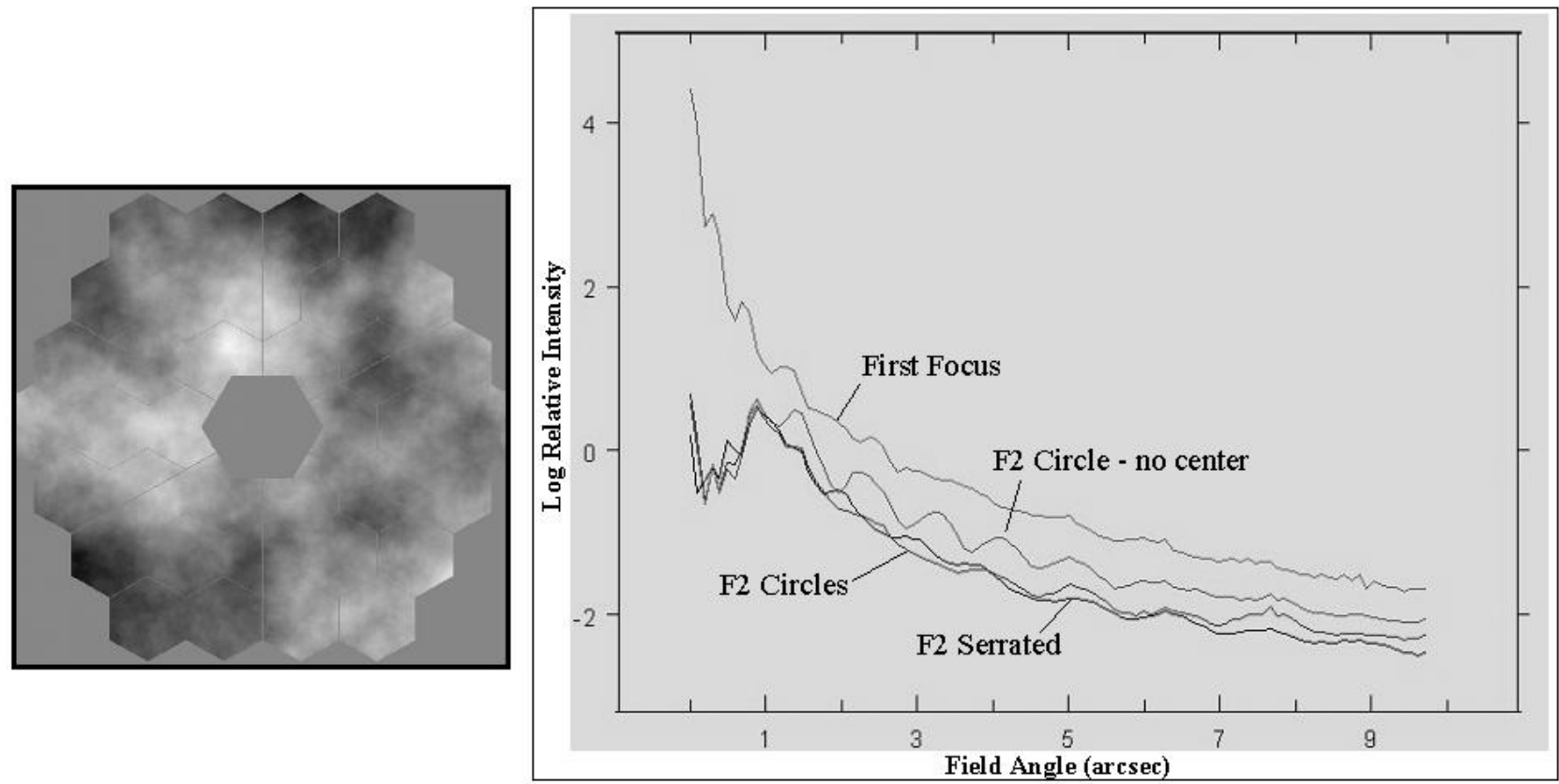

Fig. 4: The telescope pupil.

Fig. 5: Azimuthal PSF averages with and without the coronagraphic (see text). 


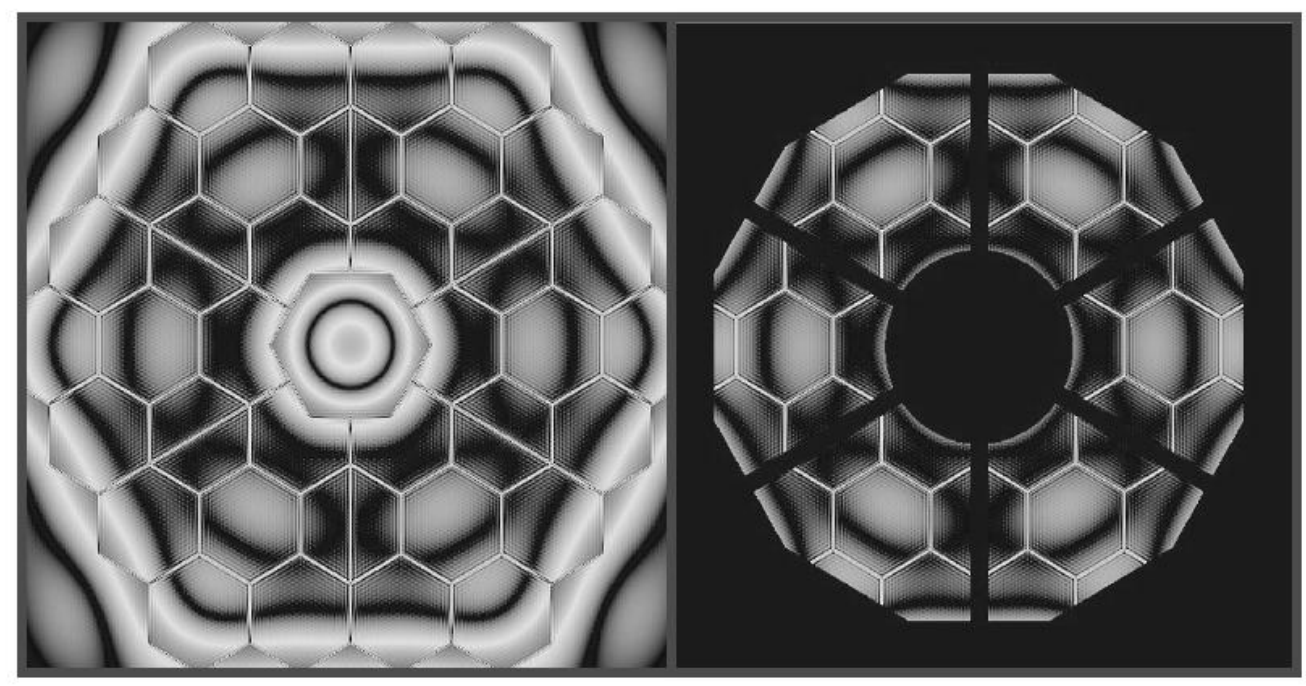

Fig. 6: Left: Pupil image with occulting spot at telescope focal plane. Right: Rotating mask applied to pupil image.

The basic design consists of: (1) a hard-edged (top hat), low-reflectivity, focal-plane mask 0.83 arcsec in radius; (2) a hard-edged, rotating pupil stop with a spider mask with widths 20 times the spider-image width. The rotating pupil stop is dodecagonal (12-sided) and scaled from the input pupil so that the outer dodecagonal edge is $90 \%$ the size of the image of the original. A central hard-edged, circular mask blocks out the secondary mirror/obscuration; that mask is $140 \%$ the size of the image of the original. The total throughput of the pupil stop is $66 \%$. The purpose of this mask is to suppress the bright regions of scattered/diffracted light shown in the model image in the left-hand panel of Fig. 6, which represents the pupil image resulting from the use of the occulting disk at the telescope focal plane. The right-hand panel of Fig. 6 shows the pupil-masked image. The reason for the dodecagonal, rather than a hexagonal, mask is evident from Fig. 6. In particular, the effect of diffraction is to make the image of the central obscuration more circularly symmetric and to make the hexagonal outer pupil edge appear softer, or more rounded. The proposed masks are intended to block more fully the unwanted radiation without reducing throughput unnecessarily.

\section{EXPECTED SENSITIVITIES}

A primary characteristic of ground-based mid-infrared $(5-30 \mu \mathrm{m})$ astronomy is high background photon flux. It is the shot noise associated with this background flux, which is estimated to be $\sim 10^{9}$ photons/s/pixel for CanariCam on the GTC, that determines the system sensitivity in all modes except, possibly, the highest resolution spectroscopy. The source of this background flux is the combined thermal emission from the atmosphere, the telescope mirrors, the telescope structure and environment, and the entrance window of the cryostat that houses the camera optics and detector. Every effort is being made to minimize the thermal emission from the GTC optics and associated structures, for which the anticipated $10-\mu \mathrm{m}$ emissivities are $6 \%$ at Cassegrain focus and $8-10 \%$ at Nasmyth focus. With the assumption that CanariCam is background-noise-limited, we have calculated the imaging and spectroscopy sensitivities given in Tables 2,3 , and 4 . The tables also indicate assumed values for other system properties. All sensitivity values correspond to the source strength that can be detected in the indicated time with a signal-to-noise ratio of unity. The indicated time is the chopped (on-source plus off-source) integration time.

Based on experience at shorter wavelengths, the sensitivity of the coronagraphic mode is expected to be dominated by systematic effects. Consider the following. To detect a faint companion around an occulted star one must subtract from the program object's observed intensity profile that of a comparison star observed in the same coronagraphic configuration. This subtraction of the normalized profile can be made to an $m$ (fractional) level of accuracy (i.e., probable error equals $m$ times the intensity at the radius where the companion may be located). Experience in the near infrared suggests that $\mathrm{m}$ is approximately 0.1 . Therefore, if the coronagraphically suppressed profile is about ten times fainter than the standard (non-coronagraphic) imaging profile, the absolute error in the measured flux is about ten times 
Table 2: Estimated sensitivities for the CanariCam imaging mode

\begin{tabular}{|l|l|r|r|r|r|}
\hline Filter designation & N (wide) & NB 11.6 & Q-wide & Qa \\
\hline & Wavelength (microns) & 10.5 & 11.6 & 20.9 & 20.5 \\
\hline & Bandwidth (microns) & 5 & 0.9 & 8.8 & 1 \\
\hline & Transmission & 0.7 & 0.7 & 0.7 & 0.6 \\
\hline & & & & & \\
\hline NEFD (mJy) & per pix, 1 s & 0.3 & 0.8 & 2.7 & 3.5 \\
\hline & per pix, 1 h & 0.005 & 0.015 & 0.043 & 0.060 \\
\hline & Point source (6x6 pix), 1 s & 2 & 5 & 16 & 21 \\
\hline & Point source (6x6 pix), 1 h & 0.03 & 0.09 & 0.26 & 0.34 \\
\hline & & & & & \\
\hline Window & Material & ZnSe-BBAR & ZnSe-BBAR & $\mathrm{KBr}$ & $\mathrm{KBr}$ \\
\hline & Transmission & 0.97 & 0.97 & 0.92 & 0.92 \\
\hline & Emissivity & 0.01 & 0.01 & 0.001 & 0.001 \\
\hline Atmosphere & Transmission & & & & \\
\hline & Emissivity & 0.98 & 0.99 & 0.5 & 0.8 \\
\hline
\end{tabular}

Table 3: Estimated sensitivities for the CanariCam10- $\mu \mathrm{m}$ spectroscopic mode

\begin{tabular}{|l|l|r|r|}
\hline Grating & & Lo-Res 10 & Hi-Res 10 \\
\hline Slit Width w(arcsec) & & 0.28 & 0.28 \\
\hline & & 0.19 & 0.67 \\
\hline NEFD (mJy) & per pix, 1h chopped & 0.9 & 3.1 \\
\hline & Point source (w x 0.48"), 1h & & \\
\hline & & $1.4 \mathrm{E}-18$ & $6.6 \mathrm{E}-19$ \\
\hline Line Flux (W/m^2) & Point source (w x 0.48"), 1h & & \\
\hline & & 10.50 & 10.50 \\
\hline Setting & Wavelength (microns) & 175 & 1313 \\
\hline & Resolution R & 0.58 & 0.58 \\
\hline & Grating/Slit Throughput & & \\
\hline Window & & 0.97 & 0.97 \\
\hline & Transmission & 0.01 & 0.01 \\
\hline Atmosphere & Emissivity & & \\
\hline & & 0.98 & 0.98 \\
\hline & Transmission & 0.02 & 0.02 \\
\hline
\end{tabular}


Table 4: Estimated sensitivities for the CanariCam 20- $\mu \mathrm{m}$ spectroscopic mode.

\begin{tabular}{|l|l|r|r|}
\hline Grating & & Lo-Res 20 & Hi-Res 20 \\
\hline Slit Width w(arcsec) & & 0.53 & 0.53 \\
\hline & & & \\
\hline NEFD (mJy) & per pix, 1h chopped & 0.83 & 2.30 \\
\hline & Point source (w x 0.96"), 1h & 7.4 & 20.5 \\
\hline Line Flux (W/m^2) & Point source (w x 0.96"), 1h & $9.0 \mathrm{E}-18$ & $3.4 \mathrm{E}-18$ \\
\hline & & & 20.50 \\
\hline Setting & Wavelength (microns) & 20.50 & 890 \\
\hline & Resolution R & 120 & 0.64 \\
\hline & Grating/Slit Throughput & 0.59 & \\
\hline Window & & & 0.92 \\
\hline & Transmission & 0.92 & 0.01 \\
\hline & Emissivity & 0.001 & 0.7 \\
\hline Atmosphere & Transmission & 0.7 & 0.3 \\
\hline & Emissivity & 0.3 & \\
\hline
\end{tabular}

smaller than for standard imaging. The integration time is then set so that the thermal-background shot noise is small compared to the absolute error fixed by $m$. For example, assuming that $\mathrm{m}=0.1$, the detection limit for an object located10 AU (1 arcsec) from a Sun-like star $10 \mathrm{pc}$ away is 15 microJanskys in coronagraphic mode compared to 150 microJanskys in the standard imaging mode. A chopped integration time of 4 hours on the program source plus a comparable amount of time on the profile-comparison standard would provide a 1- $\sigma$ noise level of about 30 microJanskys, a factor of two worse than the potential limit set by the coronagraphic mode, but much better that the sensitivity for faint companions set by profile subtraction in the standard imaging mode.

For a dual-beam polarimeter, the relationship between the desired signal-to-noise ratio and the accuracy in the determination of the degree of polarization is expressed by the equation $\mathrm{S} / \mathrm{N}=2^{1 / 2} / \Delta \mathrm{p}$. For example, an accuracy of $0.5 \%$ in the measurement of the degree of polarization requires a S/N per resolution element of 282:1 in the final reduced image. Table 5 gives the source strength that would provide a measurement of the polarization in the $\mathrm{N}$ band with a signal-to-noise ratio of three in 1 hour of chopped integration.

Table 5. Illustrative source flux densities for polarization measurements

\begin{tabular}{|cll|}
\hline $\mathbf{F}_{\mathbf{v}}(\mathbf{1 0} \boldsymbol{\mu m})$ & $\mathbf{P ( \% )}$ & $\mathbf{1 \sigma}(\boldsymbol{\%})$ \\
\hline $10 \mathrm{mJy}$ & 1.5 & 0.5 \\
\hline $50 \mathrm{mJy}$ & 0.3 & 0.1 \\
\hline $165 \mathrm{mJy}$ & 0.10 & 0.03 \\
\hline
\end{tabular}




\section{REFERENCES}

1. C. M. Telesco, R. K. Piña, K. T. Hanna, J. A. Julian, D. B. Hon, and T. M. Kisko, "GatirCam: the Gemini midinfrared imager," Proc. of the SPIE 3354, pp. 534-544, 1998.

*telesco@astro.ufl.edu; phone 1352 392-2052, ext. 265; fax 1352 392-5089; www.astro.ufl.edu; Department of Astronomy, University of Florida, 211 Bryant Space Science Center, PO Box 112055, Gainesville, FL USA 32611-2055 